corpuscles. They are round, oval, or irregularly polygonal, and sometimes show a central or eccentric oval nucleus in the shape of a light spot. The bacilli are to be found in them singly or in groups of from two to nine or more, sometimes intertwined, or, again, in irregular confusion. The cells containing bacilli occur sparingly in the midst of the infiltration, more often on the borders of the same in the apparently normal tissue immediately aljacent. I have observed them in papules embedded between the prickle cells of the rete Malpighii, and also in the initial lesion in the lumen of a large lymphatic duct. It seems to me to be beyond doubt that they possess the power of active locomotion-migratory cells. To obtain these results I had to employ a new method. Starting with the idea that bleaching measures would be serviceable in microscopic work, as well as in the arts and trade, I succeeded, after numerous fruitless attempts, in finding what I sought in permanganate of potash and sulphurous acid. In brief the method is as follows. Sections, which have been hardened in alcohol, are coloured in a solution of gentian violet (Fhrlich-Weigert), ${ }^{3}$ from twelve to twenty-four hours at the ordinary temperature, and then for two hours at a temperature of $104^{\circ} \mathrm{F}$. The section, in order to be decolourised, is first washed in absolute alcohol for several minutes, and is then, by means of a glass or platinum needle, conveyed into a watch-glass containing about three cubic centimetres of a $1 \frac{1}{2}$ per cent. aqueous solution of permanganate of potash. In this it remains about ten seconds. As a result, a brown flocculent precipitate of the hyperoxide of manganese is formed in the fluid, and also covers the section. It is then placed in an aqueous solution of chemically pure sulphurous acid, in which it is deprived of the hyperoxide of manganese, either instantaneously or in a very short time, dependent upon the conrentration of the acid; it now appears entirely rid of its colouring matter in some places, while in others it is still deeply coloured. It is next washed in distilled water, and is again placed in the solution of the permanganate of potash, in which, as in the succeeding repetitions, it remains three or four seconds, and from which it is again conveyed to the sulphurous acid. When it appears colourless, which as a rule occurs after this procedure has been gone through three or four times, it is dehydrated in alcohol, rendered transparent in clove oil, and mounted in Canada balsam. Xylol Canada balsam is best. This method of decolourisation, to which, besides those already mentioned, the bacilli of leprosy and tuberculosis do not yield, not only permits the micro-organisms of syphilis already described to be brought to view, but is a characteristic staining reaction of them. The bacilli of syphilis, leprosy, and tuberculosis are not decolourised by my method. All other bacteria are. The bacilli of syphilis, however, are differentiated from those of leprosy and tuberculosis in that the latter are not decolourised by nitric and hydrochloric acids, whereas the bacilli of syphilis are. My method failed in the examination of splenic fever, typhoid, equinia, endocarditis ulcerosa, croupous pneumonia, various secretions of wounds, the contents of acne and scabies pustules, and also in the examination of normal tissue. I wish also to mention that my attempts to prove the presence of these micro-organisms in two chancroids were futile.

By means of the method just given I have examined sixteen cases of syphilis, which I will simply enumerate without going into particulars. Sections of two initial lesions; a lymphatic gland; three papules and four products of the tertiary stage ; and further, the secretions of three initial lesions, and of the same number of moist papules. The result was a positive one in every case. The number of bacilli found varied. In general they were not very numerous. As regards the number, the age of the infiltration, and perhaps also the length of time since infection, are of importance. Thus I found in every section of two initial lesions, and of a periosteal gumma in a new-born child, one or more groups of bacilli, and here and there quite large groups among them; whereas in other cases a series of sections had to be examined before the undoubted presence of bacilli could be confirmed. According to my experience, the proof of the presence of the bacillus in syphilitic products is of equal diagnostic importance to that of the tubercle bacillus in the sputum.

Inasmuch as I have found, as a constant factor in undoubted syphilitic products of various kinds, a special kind of bacillus which differentiates itself in form and staining

3 One hundred parts of a concentrated aqueous solution of aniline with eleven parts of a concentrated alcoholic solution of gentian violet. reaction from all those known up to the present time, and which therefore is a specitic bacillus, and as we believe ourselves justified as regards other infectious diseases in assuming the constant occurrence of peculiar microorganisms to be the cause of the disease, 1 maintain, as regards syphilis, that it is very probable that the bacilli which I have discovered are the carriers of the syphilitic virus. In addition to this specially important result the above-mentioned researches present several other theoretically and practically important facts and deductions, which I will briefly mention. The occurrence of bacilli enclosed in lymphoid cells whose power of locomotion, according to my before-mentioned observation, is beyond dispute; further, the occurrence of bacilli in the apparently healthy tissue lying nearest the infiltration; and, finally, and most especially, their discovery in the initial lesion in a closed lymph-duct-support the theory that the poison of syphilis is conveyed from the place of infection, first by the lymphatic system, and later through it into the bloodvessels. The occurrence of bacilli in the rete Malpighii is of great theoretical interest, and accords with the clinical observation that in certain cases syphilitic infiltrations need only lose their covering of horny epidermis (moist papules) to be contagious.

As I have shown bacilli of the same nature as in primary and secondary products to exist in typical gummata, we must look upon the latter, as indeed do the greater number of syphilologists, as specific syphilitic affections. Only a few writers have evaded the difficulty, inasmuch as they consider gummata to be the result of a specific cachexia, and not of a syphilitic virus, basing their claim upon a number of negative inoculations, and pointing out that syphilitic patients in the tertiary stage, as a rule, produce healthy-i.e., nonsyphilitic-children. Recall to mind how long Ricord and his followers maintained the non-infectiousness of the products of the secondary stage, where the circumstances were so much simpler, because of the much greater frequency, the situation, and the nature of the manifestations Further, I cannot understand why because one has some local syphilitic deposit he should not have healthy generative cells, and as a result should not produce healthy children. In heredity we must not forget that there are affections of the generative glands which have, perhaps, no subjective or clinical symptoms, and yet are followed by the production of diseased generative cells, or, at least, of spermatic fluid containing the virus. I know from a personal communication of Armauer Hansen, and have been in a position to convince myself of it, that the testes of leprosy sufferers contain numerous bacilli in and between the seminal ducts, whether symptoms of disease of the testicles exist during life or not. In the future the question as to whether a given case is syphilis can only be affirmatively answered if the presence of the specific bacillus can be demonstrated.

In conclusion, I venture to hope that by continuous research, particularly in the cultivation and inoculation of the bacillus which I have discovered, etiological relation between it and syphilis will be so decidedly proved that none will deny it, and that a means will thereby be attained for successfully combating one of the saddest diseases of the human race. The experiences of the last few years in the successful attenuation of the virus of splenic fever and other infectious diseases warrant the hope that this expectation is not orer-sanguine.

\section{CLINICAL MEMORANDA OF TWO CASES OF CHRONIC VAGINITIS.} WITH REMARKS.

\section{BY J. BRAXTON HICKS, M.D. LOND., F.R.S., \&c.}

CAsE 1. Vagina throughout covered with granulations; freely bleeding; complete recovery.-A widow, of about sixty years, applied to me in consequence of a constant bloody discharge from the vagina. To the finger it felt velvety and granular, from os to vulva, bleeding on examination. The passage of the speculum caused much distress, and also bleeding; and it revealed the fact that from one end to the other the vagina looked as if covered by granulations as of a healing wound. The bleeding being severe, it was necessary to swab the whole surface over with tincture of perchlo- 
ride of iron diluted with three or four times its weight of water. This restrained the bleeding, but gave some pain. Astringent injections of tannin were used daily, and every third day the speculum was passed, and a sponge saturated with the dilute tincture of perchloride of iron left behind, and subsequently withdrawn. As this gave much distress, injections of the same solution were used alternately with tannin solution (two drachms to the half pint.) The vagina was very sensitive, and all manipulations were attended with distress. But at the end of a month she was free from the bleeding, and in a few weeks more the vagina had resumed its normal state.

CASE 2. Severe chronic vaginitis, with constant and free secretion of pus for over a year; treatment by injections hitherto useless; cured.--This case, though not raw and bleeding like that just described, was with this exception the most severe of any I have seen. Throughout the whole passage it was felt to be granular and velvety, and through the speculum the mucous membrane was observed pouring forth purulent secretion in large quantity. Her age was about thirty, married. The condition had come on gradually, and there was no information leading one to suspect it to have had its origin in gonorrhoa. She had been under treatment by injections for a year before I saw her. She was admitted under my care into Guy's Hospital, and the vagina treated by means of the speculum. Various applications were used in succession: nitrate of silver (twenty grains to the ounce) once; swabbing with the iron solution, as in the former case; plugging with cotton having powdered tannin diffused in it every day for some time-all of which gave gradual improvement. After a fortnight I applied a drachm of anhydrous sulphate of zinc to the upper half of the vagina, plugging it below with cotton well greased. This caused considerable pain and some exfoliation of the vagina; but when it had passed over, the vagina made a quick recovery, aided of course by continued though milder injections, till she left the hospital cured in about a month from admission.

It is not often that cases of like severity are seen; but many present themselves of long standing, with a granular state of the vagina and pus-like discharge, defying the former treatment. But these are obstinate only for the reason that they have not been thoroughly and perseveringly treated; either the syringing of the lotion has been inefficient, applied in vertical posture, or by a sponge only to the vulva, it having never properly reached the upper portion of the vagina. The mode of application is of more importance than the kind of drug employed. The material should be in contact with the diseased membrane for ten minutes at the very least, if possible for an hour, or, better still, for twelve hours; if used as an injection, the patient should be in the horizontal position. But it is best in all cases to commence treatment with a good swabbing of the upper half of the vagina with strong carbolic acid or iodised phenol through the speculum,"which should be retained for five minutes, and then the superfluous portion removed by dry cotton. Care should be taken that a portion be not drawn down to the vulva; if it should be, pain will ensue; this, however, can be removed by washing the part with wet cotton. Care also must be taken lest the carbolic acid run down the speculum on to the thigh or drawers, for if it does, a blister will ensue, and much grumbling likewise. This carbolic application might be repeated in about six or seven days, whilst twice every day an astringent lotion can be employed as strong as can be borne without distress. Of these there are numerous kinds:--Chloralum solution (one ounce to the half pint) tannin (two drachms to the half pint), boroglyceride (on ounce to the half pint), the old sulphate of zine and alum lotion, tannin and alum mixed, tannin with iodoform, bichloride of mercury ( 1 in 1000). Preceding the use of either of these, it is well to employ an antiseptic douche, in order to wash away the secretions which would impede the action of the astringents. But I prefer to apply every three or four days, through the speculum, a stronger astringent than can be employed by the syringe--such as the tincture of muriate of iron diluted three times, stron chloralum; or, as recommended by Dr. Oldham, the walls of the ragina can be kept apart by a plug of cotton. An excellent way of effecting this is by using pledgets of cotton with a string attached, diffusing through them dry powdered tannin and alum with a little iodoform, passing these through the speculum and leaving them behind, and removing them after twelve hours. This can be done every second or third day. But $I$ think that although the healthy vagina tolerates anhydrous sulphate of zinc and the tincture of muriate of iron or the weaker solution, yet when it is abraded or spongy these agents are apt to cause exfoliation of too severe a kind. The ordinary sulphate of zine or alum, however, it will readily bear. These and similar expedients will, if continued steadily, almost always cure the condition as far as the vagina is concerned; but inasmuch as the mucous membrane of the uterus is often involved, attention must be directed to this part, or the vaginitis will recur.

George-street, Hanover-square, W.

\section{MALT AS FOOD.}

\section{Bx J. MILNER FOTHERGILL, M.D.}

MalT as food has a great future before it. In the process of malting (which is a rapid germination) a distinct change is brought about in the seed. By the action of the diastase the insoluble starch is converted into soluble dextrine, which goes sweet in the mouth, so near maltose is it. The malt-combings, which are too rich in albuminoids for the brewer's purposes, are useful when malt is looked at as a food. Malt contains dextrine (some diastase), soluble albuminoids and phosphates, and when ground is an admirable food, while it is not expensive. Its taste is pleasant. It can be made into a pudding with an egg and some milk, and as such is palatable. It can be made a constituent of a milk pudding with advantage. When the brewer mashes his malt with hot water the diastase remaining in the malt converts the dextrine into maltose, which the yeast (next added) breaks up into alcohol and carbonic acid gas. The best white malt is not heated to more than $190^{\circ}$, as the diastase is acted upon injuriously by a heat above that point. Maltose is a sugar which does not readily undergo the acetous fermentation, and, therefore, as Dr. Mitchell Bruce points out in his "Materia Medica and Therapeutics," "will not give rise to acidity and dyspepsia." This is a great matter, as the cane sugar the cook adds to stewed fruit and milk puddings readily undergoes the acetous fermentation in many stomachs. The lævulose sugar of fruit, like maltose, readily undergoes the alcoholic but not the acetous fermentation. Maltose being less powerfully sweet than cane sugar, a greater quantity is necessary to sweeten the pudding. If the raw starch, semolina, sago, or tapioca be first put in the dish by itself, and placed in the oven for an hour (taking care not to lave it burnt by the oven being too hot), not only are the starch cells cracked, but a certain conversion of the starch into dextrine takes place. If to this be then added an equal quantity of ground malt and some hot milk poured on, and the dish be allowed to stand a few minutes before being put into the oven again, the diastase of the malt acts upon the farina and converts it into dextrine and maltose. Dextrine and maltose being soluble, the pudding is very thin. Such a pudding is admirably adapted for invalids and dyspeptics, as requiring scarcely any digestion in the body. For those with whom ordinary milk puddings produce acidity, such a pudding is specially suitable. Ground malt may be added to fresh milk, and forms an admirable food in cases of acute disease. Baked flour perhaps goes better with meat broths, to which it gives a high food value. (Well-baked flour requires but a touch of saliva to render it soluble, and, added to meat broths and gravy soups, renders them very nutritive.) Malt, being sweet, goes better with milk, or apple-water, or tamarındwater, or lemonade, and gives us a food which being all but independent of the digestive act, can be most usefully employed in the sick room. Beef-tea (which alone is scarcely a food) and milk-and-seltzerwater pall upon the palate of the sick person, who craves variety just as do healthy persons. The adoption of ground malt as a food will solve for us one or two knotty questions connected with feeding people when the digestive power is feeble. Drinks like lemonade, made with malt instead of cane-sugar, would not only not go sour in the mouth and stomach, but would contain some phosphates and soluble albuminoirs, and so form admirable beverages in pyrexial states. The many malt extracts now in the market are well adapted for such end. Park-street, W. 